\title{
Resistive-switching tunability with size-dependent all-inorganic zero-dimensional tetrahedrite quantum dots
}

\author{
Zhiqing Wang ${ }^{1 \dagger}$, Yueli $\mathrm{Liu}^{2 \dagger}$, Jie Shen ${ }^{1}$, Wen $\mathrm{Chen}^{1}$, Jun Miao ${ }^{3}, \mathrm{Ang} \mathrm{Li}^{1}, \mathrm{Ke} \mathrm{Liu}^{1}$ and Jing Zhou ${ }^{1 *}$
}

\begin{abstract}
All-inorganic zero-dimensional (0D) tetrahedrite $\left(\mathrm{Cu}_{12} \mathrm{Sb}_{4} \mathrm{~S}_{13}\right.$, CAS) quantum dots (QDs) have attracted extensive attention due to their excellent optical properties, bandgap tunability, and carrier mobility. In this paper, various sized CAS QDs $(5.1,6.7$, and $7.9 \mathrm{~nm})$ are applied as a switching layer with the structure $\mathrm{F}: \mathrm{SnO}_{2}$ (FTO)/CAS QDs/Au, and in doing so, the nonvolatile resistive-switching behavior of electronics based on CAS QDs is reported. The SET/RESET voltage tunability with size dependency is observed for memory devices based on CAS QDs for the first time. Results suggest that differently sized CAS QDs result in different band structures and the regulation of the SET/RESET voltage occurs simply and effectively due to the uniform size of the CAS QDs. Moreover, the presented memory devices have reliable bipolar resistive-switching properties, a resistance (ON/OFF) ratio larger than $10^{4}$, high reproducibility, and good data retention ability. After $1.4 \times 10^{6} \mathrm{~s}$ of stability testing and $10^{4}$ cycles of quick read tests, the change rate of the $\mathrm{ON} / \mathrm{OFF}$ ratio is smaller than $\mathbf{0 . 1 \%}$. Furthermore, resistiveswitching stability can be improved by ensuring a uniform particle size for the CAS QDs. The theoretical calculations suggest that the space-charge-limited currents (SCLCs), which are functioned by $\mathrm{Cu} 3 \mathrm{~d}, \mathrm{Cu} 3 \mathrm{p}$ and $\mathrm{S} 3 \mathrm{p}$ to act as electron selftrapping centers due to their quantum confinement and form conduction pathways under an electric field, are responsible for the resistive-switching effect. This paper demonstrates that CAS QDs are promising as a novel resistive-switching material in memory devices and can be used to facilitate the application of next-generation nonvolatile memory.
\end{abstract}

Keywords: memory device, tetrahedrite quantum dots, resistiveswitching tunability, resistance mechanism

\section{INTRODUCTION}

All-inorganic colloidal semiconductor quantum dots (QDs) have attracted great attention in the past decade $[1,2]$, and its most interesting aspects are their optical and electronic properties. Fortunately, these properties can be easily tuned by their sizes, phases, and components, which themselves can be precisely controlled during the formation process [3-5]. All-inorganic colloidal semiconductor QDs have great potential as active absorbers and hole transporting materials in photovoltaic devices, optoelectronics, gas sensors, and memory devices [6-9].

Focusing on memory devices, miniaturization, and intelligence, the advent of the big data era has led to the discovery of improved nonvolatile memory devices [10]. Resistive random-access memory (RRAM) is a promising technology for next-generation nonvolatile storage [11]. To date, a large number of materials, such as three-dimensional bulk oxides, nitrides, tellurides, and selenides, as well as two-dimensional (2D) thin films, have been applied as switching layers [12]. However, their complex preparation processes result in high cost and excessive power consumption. Compared with bulk materials and 2D thin films, low-dimensional inorganic materials, including zero-dimensional (0D) QDs or nanocrystals (NCs) [13-14], one-dimensional (1D) nanotubes, 1D nanowires, and 2D nanosheets [15-16], can be simply and efficiently synthesized. Furthermore, nano-memories with ultrahigh density are also preferred due to their high-speed operation, excellent tunability, small device size, and excellent resistive switching on the nanoscale

\footnotetext{
${ }^{1}$ State Key Laboratory of Advanced Technology for Materials Synthesis and Processing, School of Materials Science and Engineering, Wuhan University of Technology, Wuhan 430070, China

${ }^{2}$ State Key Laboratory of Silicate Materials for Architectures, School of Materials Science and Engineering, Wuhan University of Technology, Wuhan 430070, China

${ }^{3}$ School of Materials Science and Engineering, University of Science and Technology Beijing, Beijing 100083, China

† These authors contributed equally to this study and share the first authorship.

*Corresponding author (email: zhoujing@whut.edu.cn)
} 
structure [17].

Among these nanomaterials, memory devices based on all-inorganic QDs have several advantages, such as the ability to act as electron self-trapping centers due to outstanding quantum confinement, ambipolar charge transport, and edge effects, as well as the ability to be precisely controlled by adjusting their thickness. [3,18,19]. Graphene QDs [20], molybdenum disulfide QDs [21], black phosphorus QDs [22,23], MXene QDs [24], and perovskite QDs can be used to fabricate RRAM cells [25]. In the conventional and effective strategy of realizing resistive switching, low-dimensional inorganic materials are introduced into a polymer matrix, thereby forming an organic-inorganic hybrid structure to achieve charge trapping for the memory effect [26]. However, uniformly dispersing inorganic materials in thin films is difficult. The complex structures and properties of the active layer and polymer matrix, as well as the interface between the active layer and electrodes, result in the resistive-switching characteristics being difficult to control. To overcome this, only all-inorganic QDs should be used as the switching materials.

Recently, all-inorganic QD-based RRAM devices have attracted an increased amount of attention due to their outstanding resistive-switching characteristics, with some scholars comparing them with organic-inorganic hybrid memory devices to demonstrate their advantages. Current state-of-the-art all-inorganic QD RRAMs are based on perovskite QDs, which have a low reading voltage $(-0.3 \mathrm{~V})$ and a high light-assisted resistance (ON/OFF) ratio $\left(10^{7}\right)$ [27]. However, for perovskite QDs, unavoidable defects, such as the existence of photogenerated carriers and surface recombination charges, result in an unstable switching performance. Memory devices using Ag-In-Zn-S NCs exhibit bipolar resistance behavior across 70 endurance cycles with an ON/OFF ratio of 18 [28], which should be low for the successful commercialization of the memory devices. In light of this information, it is evident that large differences exist in the resistive-switching properties of different switching materials. Unfortunately, the intrinsic mechanism behind the resistive-switching behavior of all-inorganic QDbased RRAM devices is yet to be determined, though some mechanisms have been suggested, such as filamentary conduction $[29,30]$ and charge trapping and detrapping [31]. Therefore, extensive studies should be conducted concerning the resistive-switching properties of memory devices based on all-inorganic QDs, especially with respect to developing new switching materials and establishing an intrinsic mechanism to provide a practical solution for commercialization.

Tetrahedrite $\left(\mathrm{Cu}_{12} \mathrm{Sb}_{4} \mathrm{~S}_{13}\right.$, CAS) QDs have excellent optical properties and bandgap tunability and can be synthesized by the facile method [32,33]. As typical ptype direct bandgap semiconductors, CAS QDs have two non-occupied states in the valence band, which contributes to their excellent hole mobility and makes them a potential and novel resistive-switching material for RRAM devices [33]. However, research in this area is lacking. In our previous work, the quantum size effects of CAS QDs with bandgap tunability were reported for the first time [34,35]; we also applied CAS QDs as a charge transporting material in gas sensors and photovoltaic devices [36-38]. The previous studies provide a foundation to examine the influence of size effects and band structures on the resistive-switching behavior by designing differently sized CAS QDs.

In this work, we focus on CAS QD-based memory devices, including resistive-switching characteristics, stability, reproducibility, and the intrinsic mechanism behind their operation. CAS QDs with different sizes were designed to investigate the influence of size on the threshold voltage. Density functional theory (DFT) in combination with the nonequilibrium Green's function (NEGF) method is implemented to study the carrier transport properties of CAS QDs. Moreover, currentvoltage $(I-V)$ measurements are recorded to investigate the resistive-switching and retention properties of memristive devices. Based on the theoretical results and $I-V$ fitting curves, a detailed intrinsic mechanism behind QD resistive-switching memory devices is obtained. These observations provide important information for nextgeneration nonvolatile memory application.

\section{EXPERIMENTAL SECTION}

\section{Synthesis of tetrahedrite QDs}

Analytical grades of cuprous iodide (CuI, 99\%), oleylamine (OLA, 80\%-90\%), diphenyl ether $\left(\mathrm{C}_{12} \mathrm{H}_{10} \mathrm{O},>99 \%\right)$, $\mathrm{N}, \mathrm{N}$-diphenyl thiourea $\left(\mathrm{C}_{13} \mathrm{H}_{12} \mathrm{~N}_{2} \mathrm{~S}, 98 \%\right)$, and antimony trichloride $\left(\mathrm{SbCl}_{3}, 99 \%\right)$ were obtained from Aladdin. CAS QDs were prepared by a hot injection method. In this case, OLA was selected as the reaction solvent. We observed that, at reaction temperatures below $110^{\circ} \mathrm{C}$, OLA did not coordinate well with the metal salt, and the stability of the QD solution reduced dramatically at reaction temperatures above $150^{\circ} \mathrm{C}$. Therefore, we tested target reaction temperatures of 110,130 and $150^{\circ} \mathrm{C}$. The reaction mixture with $\mathrm{CuI}(0.2857 \mathrm{~g})$ and OLA $(30 \mathrm{~mL})$ was prepared by stirring and heating at $120^{\circ} \mathrm{C}$ in three- 
neck round-bottom flasks. The flasks were evacuated and kept under argon gas throughout the reaction. The reaction temperature was then raised to $130^{\circ} \mathrm{C}$, and the reaction mixture was aged for $10 \mathrm{~min}$, after which $\mathrm{SbCl}_{3}$ $(0.114 \mathrm{~g})$ was added and the solution was stirred continuously for $3 \mathrm{~min}$. In a separate flask, $\mathrm{C}_{13} \mathrm{H}_{12} \mathrm{~N}_{2} \mathrm{~S}$ $(0.5137 \mathrm{~g})$ was added to $2.25 \mathrm{~mL}$ of $\mathrm{C}_{12} \mathrm{H}_{10} \mathrm{O}$; the solution was then heated at $90^{\circ} \mathrm{C}$ until dissolving took place. The $\mathrm{C}_{13} \mathrm{H}_{12} \mathrm{~N}_{2} \mathrm{~S}-\mathrm{C}_{12} \mathrm{H}_{10} \mathrm{O}$ solution was rapidly injected into OLA using a quartz syringe, and the reaction process was precisely controlled at $130^{\circ} \mathrm{C}$ for $600 \mathrm{~s}$. The resultant solution was rapidly cooled to room temperature after reaction completion. Washing and centrifugation were performed to obtain purified CAS QDs, which were then dispersed in hexane for storage in a nitrogen glovebox.

\section{Device fabrication}

The fluorine-doped tin oxide (FTO)-coated glass substrate $\left(2 \times 2 \mathrm{~cm}^{2}, 15 \Omega / \square\right)$ was cleaned by ultrasound for 15 min with acetone, deionized water, and ethanol (in said order). It was then dried with a stream of pressurized nitrogen and treated with oxygen plasma for $10 \mathrm{~min}$. The CAS QD mother liquor $(1 \mathrm{~mL})$ was mixed with methanol $(3 \mathrm{~mL})$, and the precipitates were separated by centrifugation at $8000 \mathrm{rpm}$ for $3 \mathrm{~min}$. The resultant colloidal CAS QDs were then dissolved in 1-hexanethiol at a concentration of $10 \mathrm{mg} \mathrm{mL}^{-1}$. After the CAS QD solution was loaded on the FTO substrate by the spin coating technique, an Au electrode with a thickness of roughly $200 \mathrm{~nm}$ was deposited on the CAS QDs by radio frequency magnetic sputtering through a metal mask at a system pressure of $1 \times 10^{-5}$ Torr. The thickness of the active layer was adjusted by changing the rotation speed during the spin coating process or changing the duration of the process. The device was then annealed under a controlled air atmosphere on a hot plate at $90^{\circ} \mathrm{C}$ for $10 \mathrm{~min}$. The entire fabrication process was conducted in a nitrogen glovebox.

\section{Calculation details}

Optimizing the geometrical structure was carried out using DFT and the $\mathrm{DMol}^{3}$ package. General gradient approximation (GGA) was employed in the form of a Perdew-Burke-Ernzerh (PBE) exchange-correlation functional. We used a double-numeric quality basis set with dynamic nuclear polarization functions. A Fermi smearing of 0.005 hartree was used to accelerate convergence. The convergence thresholds were set as 0.002 hartree/ $\AA$ for the maximum force, $0.005 \AA$ for the maximum displacement, and $1.0 \times \mathrm{e}^{-5}$ hartree for the energy change. The geometrical optimization was stopped when the energy convergence was satisfied by the displacement or force criteria. The transport property calculations for the CAS QDs were conducted within the framework of DFT in combination with the NEGF. These were implemented in Materials Studio, using GGA with the exchange-correlation functional and the PBE basis set for an electron wave function. An energy cutoff of $150 \mathrm{Ry}$ was adopted to converge the grid integration of the charge density. A Monkhost-Pack k-mesh of $1 \times 1 \times 25$ was selected when calculating the total energy and transport properties. The transmission function was calculated from -1.0 to $1 \mathrm{eV}$ with a step size of $0.005 \mathrm{eV}$. The convergent criterion of total energy was set by the default value of the $\mathrm{DMol}^{3}$ code.

\section{Material characterization}

The crystal structure and microstructure properties of the CAS QDs were examined by high-resolution transmission electron microscopy (HRTEM, JEM-2100F, JEOL, Japan) and X-ray diffraction (XRD, Pert-Pro, PANalytical, Netherlands) with $\mathrm{Cu} \mathrm{Ka}$ radiation. Field-emission scanning electron microscopy (FESEM, Zeiss Ultra Plus, Zeiss, Germany) was used to observe the morphology of the materials and devices. The elemental mapping images and compositional ratios were analyzed using the energy dispersive X-ray spectroscope (EDS) attached to the FESEM (Model Inca X-Max50, Japan). Atomic force microscopy (AFM, Cypher ES, Oxford, USA) was used to observe the surface morphology, phase imaging, and image amplitude of the devices. UV-vis absorption (UV2550, Shimadzu, Japan) in the wavelength range of $300-800 \mathrm{~nm}$ was recorded to characterize the optical absorption properties of the samples. Photoluminescence (PL) spectra were measured on a Horiba Yvon Fluoromax 4 at room temperature. Valence band offset was measured by UV photoelectron spectroscopy (UPS, ESCALAB 250Xi, Thermo Fisher, USA) with $\mathrm{He}(21.22 \mathrm{eV})$ as the radiation source. The electrical characteristics of the fabricated devices were carried out using a Keithley 4200 semiconductor parameter analyzer. The pulse width and interval time were $1 \mathrm{~ms}$ in the endurance measurements.

\section{RESULTS AND DISCUSSION}

\section{Characterizing tetrahedrite QDs}

Fig. 1a-c show the HRTEM images of the CAS QDs prepared at temperatures of 110,130 and $150^{\circ} \mathrm{C}$, from which it is evident that the CAS QDs are spherical in shape and monodispersed. Moreover, the CAS QDs 

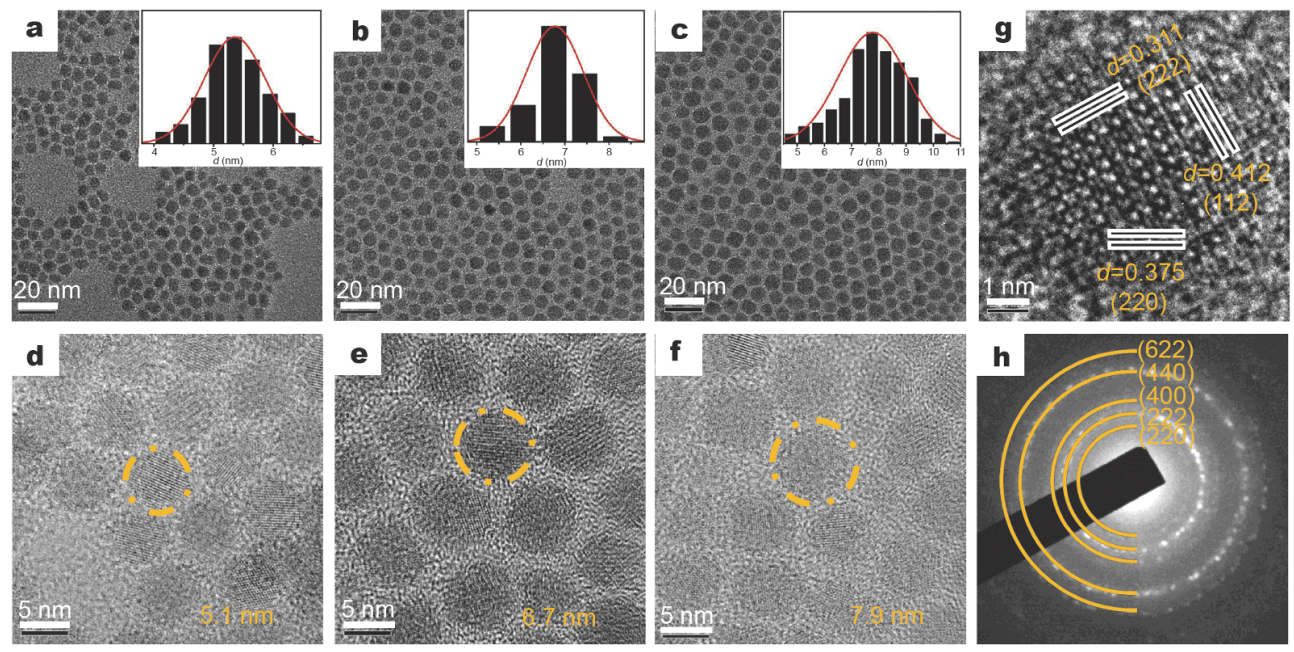

Figure 1 Characteristics of CAS QDs: low-magnification TEM images of CAS QDs at reaction temperatures of (a) $110^{\circ} \mathrm{C}$, (b) $130^{\circ} \mathrm{C}$, and (c) $150^{\circ} \mathrm{C}$. Insets are the size distribution histograms of the samples. (d-f) Local amplification images of CAS QDs from (a-c), respectively. (g) HRTEM image of a CAS QD from (d). (h) SAED pattern of the CAS QD from (g).

prepared at 110,130 and $150^{\circ} \mathrm{C}$ have diameters of $5.1 \pm$ $0.6(\sigma=9.5 \%), 6.7 \pm 0.4(\sigma=10.1 \%)$, and $7.9 \pm 0.5 \mathrm{~nm}(\sigma$ $=9.1 \%)$, respectively. Moreover, the QDs have standard deviations of about $10 \%$, which is typical for monodispersed QDs. Fig. 1d-f show the morphologies of the small $(5.1 \mathrm{~nm})$, intermediate $(6.7 \mathrm{~nm})$, and large $(7.9 \mathrm{~nm})$ QDs, from which it is evident that their sizes increase with increasing reaction temperature. Fig. $1 \mathrm{~g}$ shows the HRTEM image of a single 5.1-nm QD. The lattice image indicates it has high crystallinity. Fig. 1h shows the selected area electron diffraction (SAED) pattern, wherein the clear bright diffraction rings correspond to the (220), (222), (400), (440), and (622) reflections of the CAS QDs. Indeed, the QDs have a cubic structure. XRD was employed to determine the crystal phase of the CAS QDs (Fig. S1), the results of which are inconsistent with the results from the HRTEM images in Fig. 1g, h, wherein the
CAS QDs create a cubic lattice with a $I 43 m$ space group $(a=10.33 \AA$ ). Fig. S2 shows a simulated image of a single unit cell of the CAS QDs.

Fig. 2a and b illustrate the UV-vis spectra of the 5.1-, 6.7-, and 7.9-nm CAS QDs; the corresponding bandgaps calculated from the Tauc plots are 1.94, 1.84, and $1.76 \mathrm{eV}$, respectively. The bandgaps decrease with an increase in size, which proves that the QDs in question have excellent quantum size effects and bandgap tunability. The inset in Fig. 2a illustrates the positions of the absorption edges of the CAS QDs, from which it is evident that the absorption bands of the 5.1-, 6.7-, and 7.9-nm CAS QDs are 602, 673, and $705 \mathrm{~nm}$, respectively. When the size changes from 5.1 to $7.9 \mathrm{~nm}$, the absorption bands are red-shifted from 602 to $705 \mathrm{~nm}$. As shown in Fig. 2c, the PL emission peaks are also red-shifted from 560 to $600 \mathrm{~nm}$. The observed sizedependent bandgap tunability and the red-shifting optical
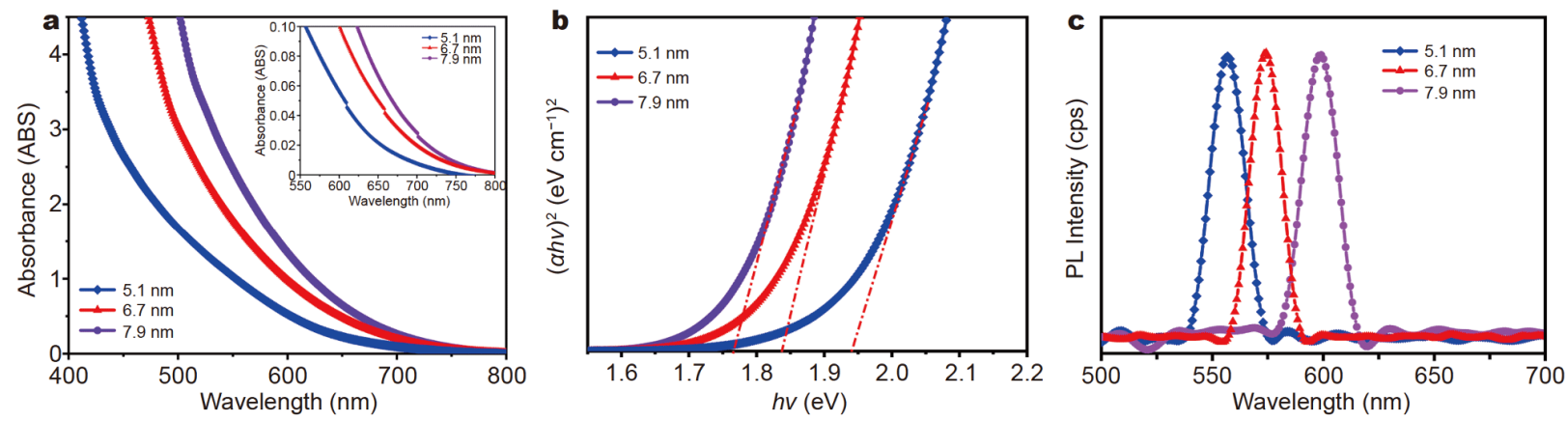

Figure 2 Optical properties of CAS QDs with different sizes: (a) UV-vis absorption spectra, (b) energy gaps calculated using the plots of $(\alpha h v)^{2} v s$. $h v$, and (c) PL spectra. 
properties are consistent with the quantum confinement effects $[3,32]$.

The scheme of the device structure is shown in Fig. 3a. The CAS QD layer and Au electrode in Fig. $3 b$ have the same thickness, i.e., $200 \mathrm{~nm}$. The top-view FESEM image indicates that the CAS QD layer completely covers the FTO glass surface (Fig. S3a, b). The surface element distribution of the CAS QD layer is revealed by the elemental mapping images shown in Fig. 3c-g. Fig. $3 \mathrm{c}$ shows the top-view SEM image of the FTO/CAS QDs, and Fig. $3 \mathrm{~d}$ shows the local amplification of the selected region. Fig. 3e-g display the corresponding mapping images of the $\mathrm{Cu}, \mathrm{Sb}$, and $\mathrm{S}$ elements, indicating that these elements are uniformly distributed in the CAS QD layer. The EDS spectrum of Fig. 3c-g is also shown in Fig. S3, the inset table of which shows that the $\mathrm{Cu}: \mathrm{Sb}: \mathrm{S}$ ratio is 12:4.3:13.2, indicating that tetrahedrite is slightly rich in antimony. Fig. S3d is the backscattered electron image corresponding to Fig. $3 \mathrm{~b}$ after more than $1.4 \times 10^{6} \mathrm{~s}$ of stability testing and $10^{4}$ cycles of reproducibility tests, from which it is evident that there is no migration of the top electrode, which means that the resistance switching behavior of the FTO/CAS QDs/Au device is dependent on the CAS QDs instead of the diffused Au atoms.

According to Fig. S4a-c, the surfaces of the CAS QD layers corresponding to the 5.1-, 6.7-, and 7.9-nm QDs on top of the FTO possess root mean square roughness values of 5.3, 3.1, and $4.3 \mathrm{~nm}$, respectively. The AFM images shown in Fig. S4d-f suggest a relatively spherical structure with respect to the CAS QDs. Moreover, the AFM amplitude images in Fig. S4g-i illustrate the surface undulation of the memory devices. Indeed, surface roughness and surface undulation are important with respect to ensuring the smooth and homogeneous coverage of CAS QDs spin-coated on the FTO substrate and prove that CAS QD size does not change after the nearly whole coverage of CAS QDs on the FTO surface and further annealing treatment in our case.

\section{Room-temperature resistive-switching properties}

Room-temperature resistive-switching properties can be investigated using the $I-V$ curves shown in Fig. $4 \mathrm{a}-\mathrm{c}$, from which it is evident that the voltage is swept in the sequence of $0 \rightarrow-2 \rightarrow 0 \rightarrow 2 \rightarrow 0 \mathrm{~V}$ during the test session. In digital memory devices, the transition from the high-resistance state (HRS; i.e., OFF state) to the lowresistance state (LRS; i.e., ON state) is equivalent to a writing process, which, in this paper, is called the SET process, wherein the threshold voltage is the SET voltage. Alternatively, the transition from the LRS to the HRS is
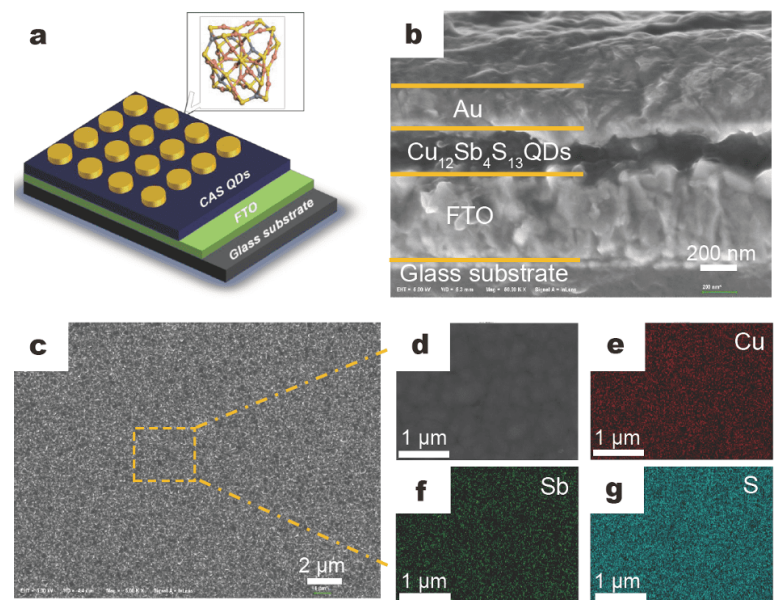

Figure 3 (a) Schematic of FTO/CAS QDs/Au memory device, (b) crosssectional FESEM image, (c) top-view SEM image, (d) local amplification of the selected region, and $(\mathrm{e}-\mathrm{g})$ element mapping images obtained by EDS.

equivalent to an erasing process, which, in this paper, is called the RESET process, wherein the threshold voltage is the RESET voltage.

We can use the $I-V$ curve of the 7.9-nm CAS QDs to describe the process. Initially, the device is in the HRS. When a negative voltage is applied, the Au electrode increases to $-0.46 \mathrm{~V}$, and the current increases rapidly from roughly $10^{-6}$ to $10^{-2} \mathrm{~A}$, indicating that the device transitions from the HRS to the LRS and that the SET voltage is $-0.46 \mathrm{~V}$. The device has good stability at the LRS in the negative (Sweep 4) and positive sweeps (Sweeps 5 and 6). Subsequently, the current decreases from roughly $10^{-2}$ to $10^{-6} \mathrm{~A}$ after $0.51 \mathrm{~V}$ (Sweep 7), suggesting that the device transits from the LRS to the HRS. The device remains in the HRS during the subsequent positive sweep (Sweep 8). The 6.7- and 5.1-nm CAS QDs have similar resistiveswitching behavior; however, differences can be seen in the SET and RESET voltages across the various sized CAS QDs. Indeed, a memory device can be maintained in the LRS by applying a positive bias of the same magnitude or turning off the power, indicating a nonvolatile memory effect.

The SET voltage was investigated for the CAS QDs using 100 memory units. Fig. S5 shows the curves obtained during the successive switching cycles. As shown in Fig. $4 \mathrm{~d}-\mathrm{f}$, the sample statistics suggest that the SET voltages for the 7.9-, 6.7-, and 5.1-nm CAS QDs are $-0.46,-1.02$, and $-1.51 \mathrm{~V}$, respectively, whereas the RESET voltages are $0.51,1.08$, and $1.61 \mathrm{~V}$, respectively. The stability of the memory device is shown in Fig. $4 \mathrm{~g}-\mathrm{i}$, from which it is evident that, for a memory device with a readout voltage of $-0.1 \mathrm{~V}$, slight fluctuations exist in the 

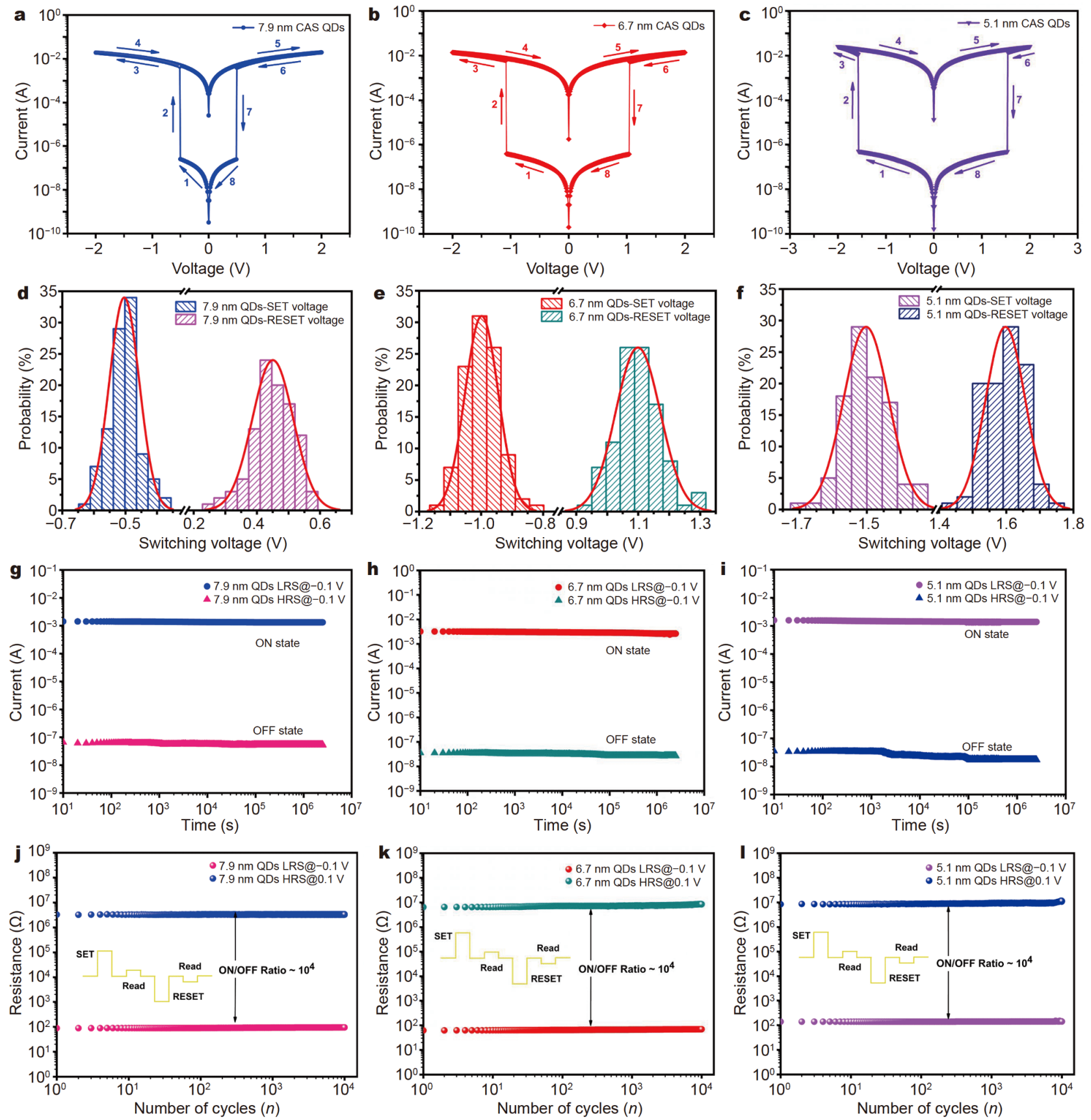

Figure 4 Resistive-switching performance of the FTO/CAS QDs/Au device: (a-c) typical resistive-switching $I-V$ characteristics in a semi-logarithm scale for the 7.9-, 6.7-, and 5.1-nm CAS QDs, respectively; (d-f) SET/RESET voltage distribution histograms; (g-i) memory device stability at -0.1 V for over $1.4 \times 10^{6} \mathrm{~s}$ of stability testing; (j-1) reproducibility of the FTO/CAS QDs/Au memory device.

HRS; however, the ON/OFF ratio still exceeds $10^{4}$ and lasts for more than $1.4 \times 10^{6} \mathrm{~s}$ without electrical degradation. The reproducibility of the memory device under a continuous read pulse is shown in Fig. $4 \mathrm{j}-\mathrm{l}$, from which it is evident that memory device in the HRS and the LRS has a voltage of 0.1 and $-0.1 \mathrm{~V}$, respectively.
Indeed, the LRS and the HRS values are almost equivalent after $10^{4}$ quick read tests, suggesting excellent device reproducibility.

The measured $I-V$ curve shows typical bipolar resistive-switching behavior and nonvolatile memory effect. In addition, the memory devices have an ON/OFF ratio 
larger than $10^{4}$, high reproducibility, and good data retention. After $1.4 \times 10^{6} \mathrm{~s}$ and $10^{4}$ cycles of quick read tests, the change rate of the ON/OFF ratio is smaller than $0.1 \%$, which is comparable to organic-inorganic hybrid memory devices and higher than some all-inorganic QDbased memory devices $[26,28]$. Therefore, CAS QDs can be used as an active layer in nonvolatile resistiveswitching memory devices. Furthermore, the observation for the SET/RESET voltage and the size dependency of the developed CAS QDs is unprecedented for RRAM devices based on 0D QDs.

\section{Electronic and transport property computations of tetrahedrite QDs}

Electronic and transport property computations were conducted using Materials Studio based on DFT and NEGF. To simplify the calculation model of electron transport properties, a transport device was constructed by sandwiching 0D CAS QDs $(d=5.1 \mathrm{~nm})$ between the $\mathrm{Au}$ (top) and FTO (bottom) electrodes (Fig. 5a). The potential of the FTO electrode was maintained at $0 \mathrm{~V}$, whereas that of the $\mathrm{Au}$ electrode was maintained at $-1.5 \mathrm{~V}$. The optimized CAS QD structure model is shown in Fig. 5b, from which it is evident that groups of tetrahedrite are contained in the CAS QDs as repeating units. Fig. $5 \mathrm{c}$ is a local enlargement of the repeating units. When voltage is applied, electrons are injected into the CAS QDs from the Au electrode. The electron trapping capability of the atoms in the CAS QDs is shown in Fig. 5d, The greater value, the stronger electron capture ability. As shown in Fig. $5 \mathrm{~d}, \mathrm{Cu}$ and $\mathrm{S}$ act as electron capture centers in the CAS QDs. Applying a bias voltage influences the bias potential, thereby enabling transmission along the scattering region of the CAS QD transport device, which can be seen from the QD transmission spectrum (Fig. S6a).

When a bias voltage is applied, a sharp maximum peak is observed in the conduction band, rather than the valence band. In this case, the applied potential is sufficient for the electrons to traverse from the valence band to the conduction band. Fig. S6b shows the calculated $I-V$ curves, from which it is evident that the current increases rapidly at $-0.35 \mathrm{~V}$. This can be explained by examining the projected density of states (PDOS) at a bias voltage of 0 and $-0.35 \mathrm{~V}$, as shown in Fig. S7a, b. According to the PDOS plots, the valence band maximums (VBMs) of the CAS QDs are mainly derived from the $\mathrm{Cu} 3 \mathrm{~d}, \mathrm{Cu} 3 \mathrm{p}$ and S $3 p$ orbitals, whereas the conduction band minimums (CBMs) are mainly derived from the $\mathrm{Cu} 4 \mathrm{p}$ and $\mathrm{Sb} 5 \mathrm{p}$ orbitals. These results suggest that, for CAS QDs with a bias voltage of $0 \mathrm{~V}$, the $\mathrm{Cu} 3 \mathrm{~d}, \mathrm{Cu} 3 \mathrm{p}$ and $\mathrm{S} 3 \mathrm{p}$ orbitals of the VBM transition are superior to the $\mathrm{Cu} 4 \mathrm{p}$ orbitals of the CBM. Fig. S6b shows the metallic behavior of the CAS QDs at a bias voltage of $-0.35 \mathrm{~V}$, indicating that the injected electrons are captured in the VBM and occupied by the $\mathrm{Cu} 3 \mathrm{~d}, \mathrm{Cu} 3 \mathrm{p}$ and S 3p orbitals [39]. Furthermore, as shown in Fig. S7c, the deformed electron density of $\mathrm{Cu}$ in
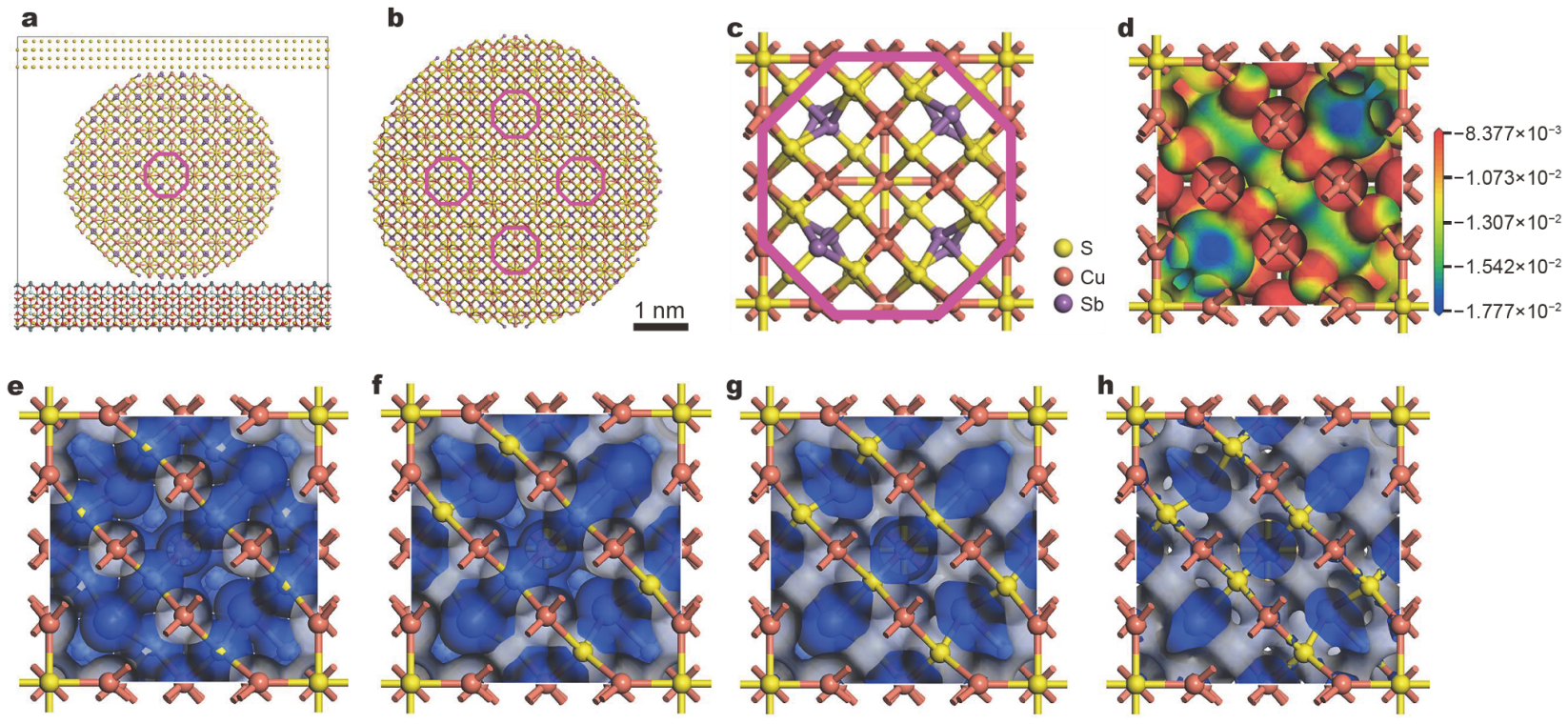

Figure 5 Electron transport properties of FTO/CAS QDs/Au electron transport devices: (a) optimized structures, (b) optimized CAS QD structure models, (c) repeating units contained in the CAS QDs, (d) the electron trapping capability of every atom, and (e-h) the process of electron capturing in the CAS QDs. 
the left and right electrodes indicates that, at a bias voltage of $-0.35 \mathrm{~V}$, many electrons are transferred from the electrode to the CAS QDs, and the injected electrons move freely between the QDs and electrode. Fig. 5e-h show the electron capture process, from which it is evident that $\mathrm{Cu}$ and $\mathrm{S}$ act as electron self-trapping centers and form conduction pathways in the memory device.

\section{Intrinsic mechanism behind the OD QD resistive-switching memory device}

To determine the switching mechanism of the memory devices, taking the 6.7-nm QDs as an example, the $I-V$ curves for the SET process were plotted in double logarithmic coordinates. Fig. 6 a shows the SET process $(0 \rightarrow$ $-2 \rightarrow 0 \mathrm{~V})$. In the HRS, the plot of $\ln I v s . \ln V$ with a slope of $\sim 1.06$ reveals a linear plot at a low voltage range, which is shown in Fig. 6b, indicating Ohmic conduction behavior for $0 \rightarrow-0.56 \mathrm{~V}$ (Step A in Fig. 6a). Two kinds of slopes can be observed. On the one hand, for the negative sweep of $-0.57 \rightarrow-0.78 \mathrm{~V}$ (Step B), a line relation can be observed in the plot of $\ln I v s$. $V^{1 / 2}$, indicating electrical conduction governed by thermionic emission (Fig. 6c). On the other hand, for the negative sweep of $-0.78 \rightarrow$
$-1.06 \mathrm{~V}$ (Steps C and D), a line relation can be observed in the plot of $\operatorname{Ln}(I) v s . \operatorname{Ln}(V)$ with a slope of $\sim 2.07$, which is consistent with the classical space-charge-limited current (SCLC) shown in Fig. 6d. In the LRS, the current has a linear plot with a slope of $\sim 1.00$, implying Ohmic conduction.

Resistive-switching properties are affected by various factors. Researchers have used defects, vacancies, interstitials, and cation substitutions formed in the resistiveswitching layer and environment during device fabrication to regulate the resistive-switching effect $[22,40,41]$. In our work, the differences in the SET/RESET voltage between the 7.9-, 6.7-, and 5.1-nm CAS QDs can be attributed to the Schottky barrier at the interface between the CAS QDs and the FTO electrode. UPS was used to explore the exact energy levels of the CAS QDs, the results of which are shown in Fig. S8. Under a bias of $10 \mathrm{eV}$, the Fermi energy $\left(E_{\mathrm{F}}\right)$ position relative to the vacuum level and the difference between the $E_{\mathrm{F}}$ and the VBM were calculated for the CAS QDs with the intercept for the secondary electron onset. In combination with the bandgaps shown in Fig. $2 \mathrm{~b}$ and the work functions of the electrodes, Fig. 7a shows the band structure alignment of
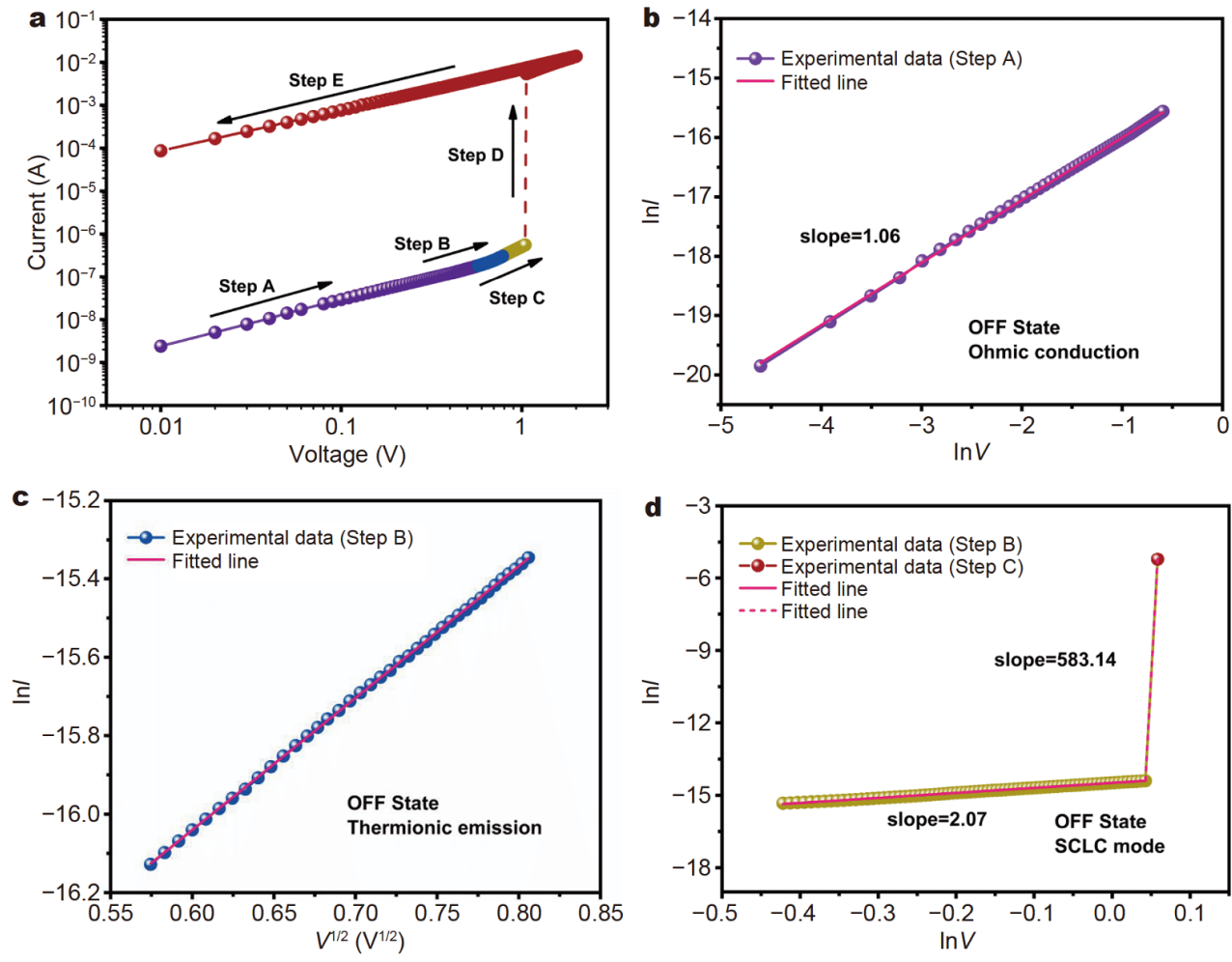

Figure 6 Experimental and fitted $I-V$ characteristics of the FTO/CAS QDs/Au device with (a) $I-V$, (b) $\ln I-\ln V$, (c) $\ln I-V^{1 / 2}$, and (d) $\ln I-\ln V$ characteristics during the SET process in a negative voltage sweep. 
the CAS QDs, from which it is evident that the aggregation of electrons increases the Schottky barrier height at the interface. Fig. S9 shows the schematics of the Schottky barrier at the interface. The height of the barrier reduces with an increase in the size of the CAS QDs. Therefore, the SET/RESET voltages are size-dependent.

In this study, a detailed mechanism is described from the perspective of the electron quantum transport theory of conductors, as well as the calculation results. For simplification, a one-trap case is considered for the modeling. Fig. $7 \mathrm{~b}$ shows a schematic of the asymmetric trap structure. The trap-filled limit voltage $\left(V_{\mathrm{TFL}}\right)$ can be determined by the trap density [42]:

$V_{\mathrm{TFL}}=\frac{e n_{t} L^{2}}{2 \varepsilon \varepsilon_{0}}$,

where $L$ denotes the thickness of the CAS QDs, $\varepsilon$ denotes a relative dielectric constant, and $\varepsilon_{0}$ denotes the vacuum permittivity of the CAS QDs. For CAS QDs, Cu 3d, Cu 3p and $S 3 p$ act as the electron capture centers. In the SET process (negative bias region), with the negative bias increasing from $0 \mathrm{~V}$, as shown in Fig. 8a, the injected electrons cannot fill the traps because, under a low operation bias voltage $\left(V_{\min }<V<V_{\mathrm{TFL}}\right)$, the reduced injected carrier concentration is less than the equilibrium concentration. At this state, the current density $(J)$ formed by the electron flow transmitted from the FTO to the CAS QDs can be expressed as follows [43]:

$J=\frac{q N_{c} V_{\mathrm{R}}}{1+\left(V_{\mathrm{R}} / V_{\mathrm{D}}\right)} \exp \left(-\frac{q \phi_{\mathrm{BN}}}{k T}\right)\left[\exp \left(\frac{q V}{k T}\right)-1\right]$,

where $V_{\mathrm{R}}$ and $V_{\mathrm{D}}$ denote the thermionic emission process and the thermionic diffusion process, respectively. For CAS QDs, $V_{\mathrm{D}}$ can be described as $V_{\mathrm{D}} \approx \mu_{\mathrm{n}} E_{\mathrm{m}}$, where $E_{\mathrm{m}}$ denotes the electric field strength and $\mu_{\mathrm{n}}$ denotes the carrier mobility, which can be defined as $\mu=e \tau / \mathrm{m}^{*}$, where $e$ denotes the electron charge, $\tau$ denotes the carrier relaxation time, and $m^{*}$ denotes the carrier effective mass, which can be calculated from $\left(m^{*}\right)^{-1}=\left(1 / \hbar^{2}\right) \partial^{2} E / \partial k^{2}\left(m^{*}\right)$, where $\hbar$ denotes the reduced Planck's constant and $E \approx k$ denotes the band structure. Therefore, for CAS QDs with two non-occupied states in the valence band, the carrier mobilities are high, which ensures that the current follows Ohmic conduction behavior due to the thermionic emission process. In turn, this causes charge injection through the energy barrier between the FTO and CAS QDs. Therefore, the device starts in the HRS and follows Ohmic conduction behavior under a low operation bias voltage $\left(V_{\min }<V<V_{\mathrm{TFL}}\right.$, State 1$)$.

With an increase in the bias voltage $\left(V_{\mathrm{TFL}}<V<V_{1}\right.$, State 2), the injected electrons gradually fill the traps; however, they cannot pass over the $h_{1}$ barrier nor reduce the active material trap density in the trap-filled SCLC region. At this stage, the current begins to increase following the near square law. With a further increase in the bias voltage $\left(V>V_{1}\right.$, State 3$)$, the trapped electrons jump over the $h_{1}$ barrier; when all of the traps are filled with the injected electrons, the injected electrons move freely by trap-to-trap hopping, thereby forming the conduction pathway. As a result, the device transits from the HRS to the LRS, and the current follows Ohmic conduction. At this point, the traps remain filled, and the device is in the LRS when the negative bias is reduced $\left(V>V_{1}\right.$, State 4$)$, which is shown in Fig. 8b.

In the RESET process (positive bias region), with an increase in the positive bias voltage to a low region $\left(V_{\min }\right.$ $<V<V_{2}$, State 5), as shown in Fig. 8c, the traps remain filled because the trapped electrons cannot be released, and the electron concentration remains at a high level, thereby ensuring the LRS state. When $V_{2}<\mathrm{V}<V_{\mathrm{TFL}}$, the
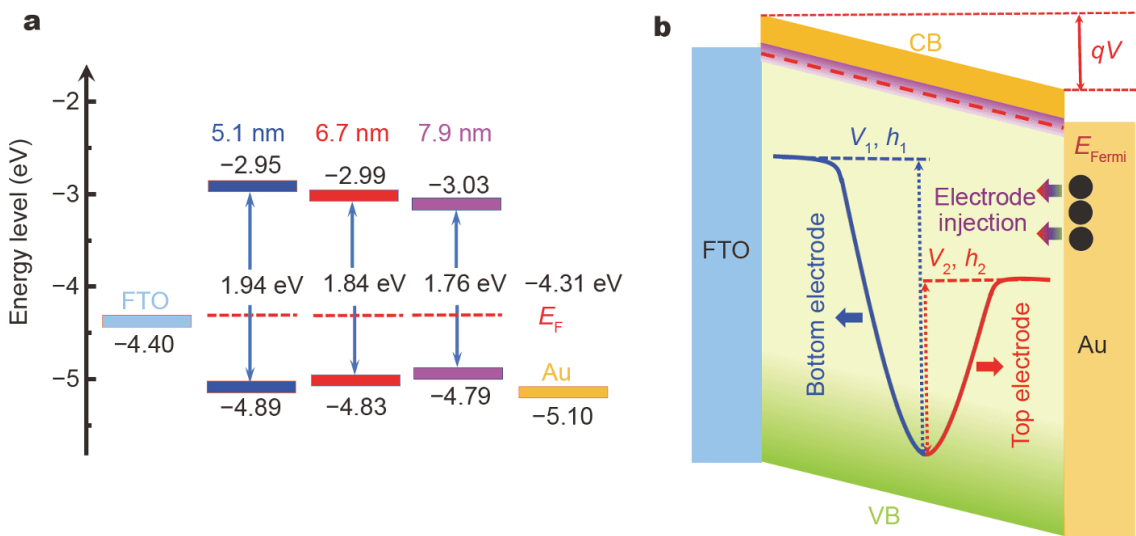

Figure 7 (a) Schematic of different sized CAS QDs for the band structure alignment and (b) schematic of the asymmetric trap structure. 

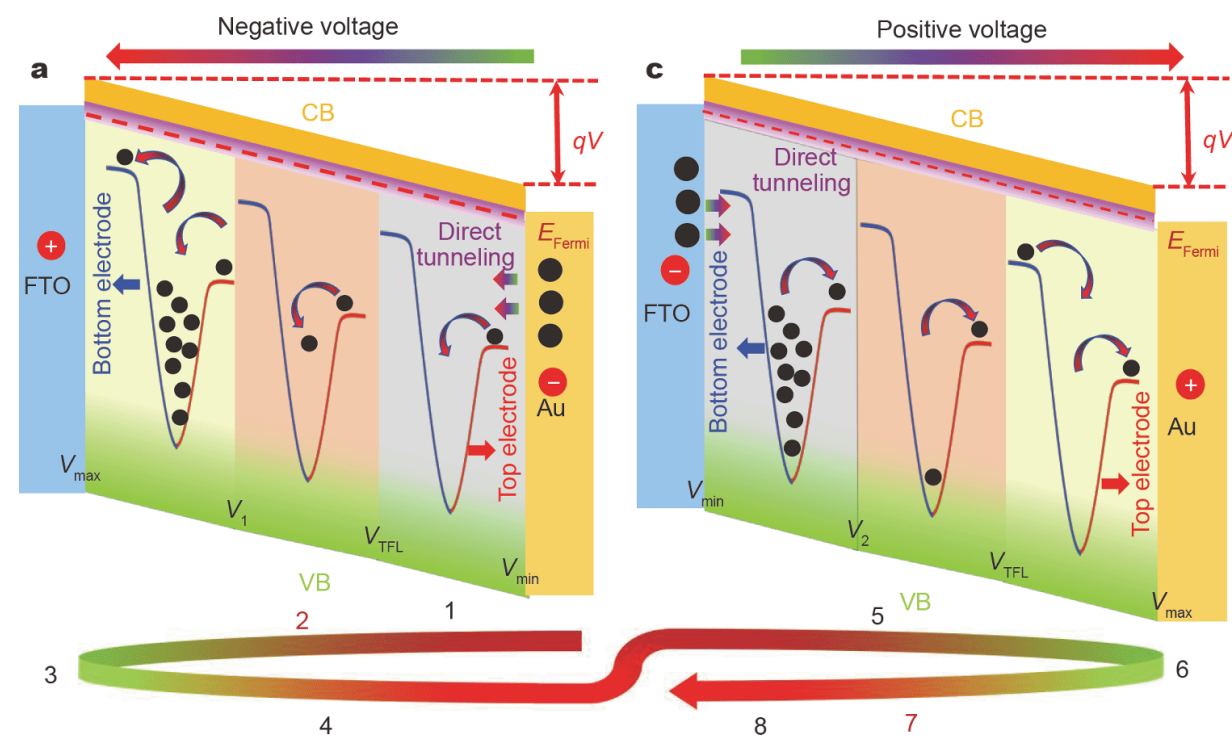

4
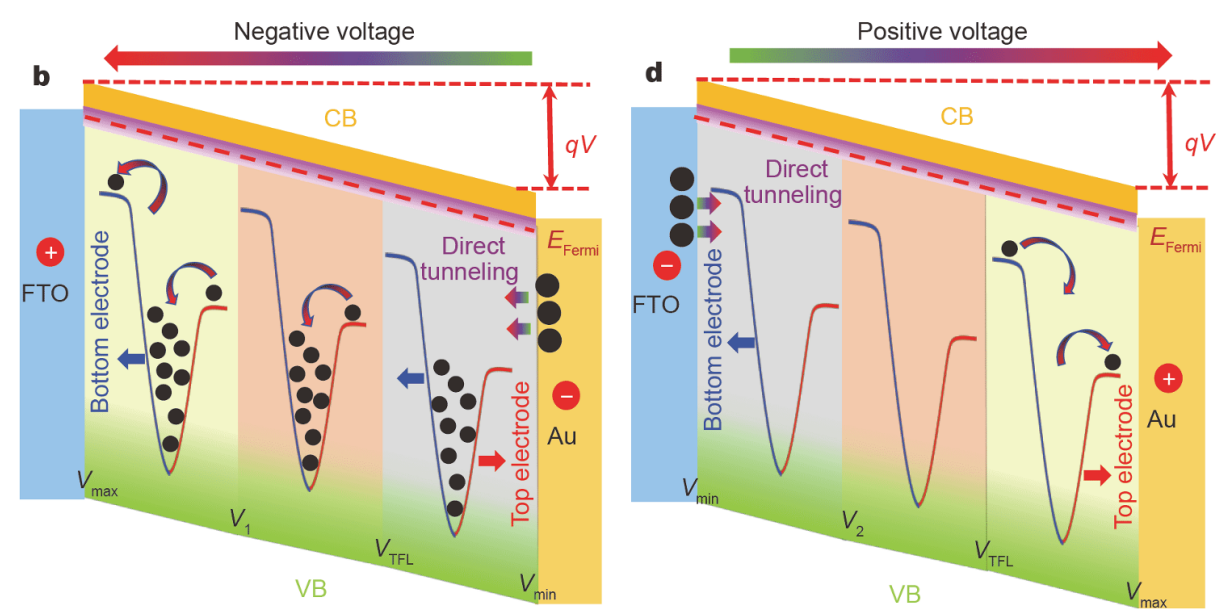

Figure 8 Schematic of detailed intrinsic mechanism for the resistive-switching behavior of the CAS QDs: (a) negative bias increasing in the SET process, (b) negative bias decreasing in the SET process, (c) positive bias increasing in the RESET process, and (d) positive bias decreasing in the RESET process.

traps release the electrons (over the barrier $h_{2}$ ) with no further electrons filling the trap, thus forming a higher electron concentration. This is known as the detrapping effect. When the positive bias surpasses $V_{\text {TFL }}$, the injected electrons once again fill the traps but cannot jump immediately over the barrier due to the large activation energy. In this state $\left(V_{\mathrm{TFL}}<V<V_{\max }\right.$, State 6), the injected electrons contribute to the current directly, though the traps are unoccupied, and the device remains in the LRS. As shown in Fig. 8d, the device changes to the HRS due to the fact that the electron concentration is smaller than the equilibrium concentration when the decreasing negative voltage is smaller than $V_{\mathrm{TFL}}\left(V_{2}<V<V_{\mathrm{TFL}}\right.$, State 7). In the following process, the device is maintained in the HRS $\left(V_{\text {min }}<V<V_{2}\right.$, State 8$)$.

\section{CONCLUSIONS}

In this paper, we demonstrated the potential application of all-inorganic CAS QDs in memory devices with resistive-switching behavior. Memory devices were fabricated with different sized CAS QDs using a simple spin coating method. The size-dependent SET/RESET voltage was observed in the CAS QD memory devices due to the quantum size effects with bandgap tunability; moreover, the small CAS QDs had high barriers at the interface between the FTO electrode and the CAS QDs. Furthermore, the devices have a reliable bipolar resistiveswitching property, an ON/OFF ratio larger than $10^{4}$, excellent stability over $1.4 \times 10^{6} \mathrm{~s}$, and excellent reproducibility after $10^{4}$ quick read tests. A detailed intrinsic mechanism behind the CAS QD memory devices 
was systematically proposed and investigated. This was further examined by DFT in combination with NEGF. The results suggest that the SCLC, which is functioned by $\mathrm{Cu} 3 \mathrm{~d}, \mathrm{Cu} 3 \mathrm{p}$ and $\mathrm{S} 3 \mathrm{p}$ to act as electron self-trapping centers due to their quantum confinement and form conduction pathways under an electric field, is responsible for the resistive-switching effect. Overall, the findings suggest that CAS QDs are a candidate with respect to the active layer in resistive-switching memory devices for next-generation nonvolatile memory designs.

\section{Received 13 February 2020; accepted 5 May 2020;} published online 3 August 2020

1 Wang Y, Li X, Song J, et al. All-inorganic colloidal perovskite quantum dots: A new class of lasing materials with favorable characteristics. Adv Mater, 2015, 27: 7101-7108

$2 \mathrm{Li} \mathrm{J}$, Xu L, Wang T, et al. 50-Fold EQE improvement up to 6.27\% of solution-processed all-inorganic perovskite $\mathrm{CsPbBr}_{3}$ QLEDs via surface ligand density control. Adv Mater, 2017, 29: 1603885

3 Kagan CR, Lifshitz E, Sargent EH, et al. Building devices from colloidal quantum dots. Science, 2016, 353: aac5523

4 Sargent EH. Colloidal quantum dot solar cells. Nat Photon, 2012, 6: $133-135$

5 Xie R, Rutherford M, Peng X. Formation of high-quality I-III-VI semiconductor nanocrystals by tuning relative reactivity of cationic precursors. J Am Chem Soc, 2009, 131: 5691-5697

6 Chen J, Liu D, Al-Marri MJ, et al. Photo-stability of $\mathrm{CsPbBr}_{3}$ perovskite quantum dots for optoelectronic application. Sci China Mater, 2016, 59: 719-727

7 Lv L, Xu Y, Fang H, et al. Generalized colloidal synthesis of highquality, two-dimensional cesium lead halide perovskite nanosheets and their applications in photodetectors. Nanoscale, 2016, 8: 13589-13596

8 Liu H, Li M, Voznyy O, et al. Physically flexible, rapid-response gas sensor based on colloidal quantum dot solids. Adv Mater, 2014, 26: 2718-2724

9 Pozner R, Lifshitz E, Peskin U. A triple quantum dot based nanoelectromechanical memory device. Appl Phys Lett, 2015, 107: 113109

10 Qian K, Nguyen VC, Chen T, et al. Novel concepts in functional resistive switching memories. J Mater Chem C, 2016, 4: 9637-9645

11 Li S, Zeng F, Chen C, et al. Synaptic plasticity and learning behaviours mimicked through $\mathrm{Ag}$ interface movement in an $\mathrm{Ag} /$ conducting polymer/Ta memristive system. J Mater Chem C, 2013, 1: 5292-5298

12 Yang JJ, Strukov DB, Stewart DR. Memristive devices for computing. Nat Nanotech, 2013, 8: 13-24

13 Zhang P, Xu B, Gao C, et al. Facile synthesis of $\mathrm{Co}_{9} \mathrm{Se}_{8}$ quantum dots as charge traps for flexible organic resistive switching memory device. ACS Appl Mater Interfaces, 2016, 8: 30336-30343

14 Kim DH, Wu C, Park DH, et al. Flexible memristive devices based on InP/ZnSe/ZnS core-multishell quantum dot nanocomposites. ACS Appl Mater Interfaces, 2018, 10: 14843-14849

15 Shi H, Zheng J, Cheng B, et al. Rewritable non-volatile stress information memory by bulk trap-induced giant piezoresistance effect in individual $\mathrm{PbS}$ micro/nanowires. J Mater Chem C, 2017, 5: $229-237$
16 Son D, Chae SI, Kim M, et al. Colloidal synthesis of uniform-sized molybdenum disulfide nanosheets for wafer-scale flexible nonvolatile memory. Adv Mater, 2016, 28: 9326-9332

17 Hong AJ, Song EB, Yu HS, et al. Graphene flash memory. ACS Nano, 2011, 5: 7812-7817

18 Terabe K, Hasegawa T, Nakayama T, et al. Quantized conductance atomic switch. Nature, 2005, 433: 47-50

19 Xue Q, Zhang $\mathrm{H}$, Zhu M, et al. Photoluminescent $\mathrm{Ti}_{3} \mathrm{C}_{2}$ MXene quantum dots for multicolor cellular imaging. Adv Mater, 2017, 29: 1604847

20 Pan X, Skafidas E. Resonant tunneling based graphene quantum dot memristors. Nanoscale, 2016, 8: 20074-20079

21 Wang D, Ji F, Chen X, et al. Quantum conductance in $\mathrm{MoS}_{2}$ quantum dots-based nonvolatile resistive memory device. Appl Phys Lett, 2017, 110: 093501

22 Han ST, Hu L, Wang X, et al. Black phosphorus quantum dots with tunable memory properties and multilevel resistive switching characteristics. Adv Sci, 2017, 4: 1600435

23 Zhang X, Xie H, Liu Z, et al. Black phosphorus quantum dots. Angew Chem Int Ed, 2015, 54: 3653-3657

24 Mao H, Gu C, Yan S, et al. MXene quantum dot/polymer hybrid structures with tunable electrical conductance and resistive switching for nonvolatile memory devices. Adv Electron Mater, 2020, 6: 1900493

25 Wang Y, Lv Z, Liao Q, et al. Synergies of electrochemical metallization and valance change in all-inorganic perovskite quantum dots for resistive switching. Adv Mater, 2018, 30: 1800327

26 Hwang B, Lee JS. Recent advances in memory devices with hybrid materials. Adv Electron Mater, 2019, 5: 1800519

27 Chen Z, Zhang Y, Yu Y, et al. Light assisted multilevel resistive switching memory devices based on all-inorganic perovskite quantum dots. Appl Phys Lett, 2019, 114: 181103

28 Zhou D, Chen F, Han S, et al. Resistive switching characteristics of AgInZnS nanoparticles. Ceramics Int, 2018, 44: S152-S155

29 Xue W, Gao S, Shang J, et al. Recent advances of quantum conductance in memristors. Adv Electron Mater, 2019, 5: 1800854

30 Huang Y, Zhao Z, Wang C, et al. Conductive metallic filaments dominate in hybrid perovskite-based memory devices. Sci China Mater, 2019, 62: 1323-1331

31 Xia Y, He W, Chen L, et al. Field-induced resistive switching based on space-charge-limited current. Appl Phys Lett, 2007, 90: 022907

32 van Embden J, Latham K, Duffy NW, et al. Near-infrared absorbing $\mathrm{Cu}_{12} \mathrm{Sb}_{4} \mathrm{~S}_{13}$ and $\mathrm{Cu}_{3} \mathrm{SbS}_{4}$ nanocrystals: Synthesis, characterization, and photoelectrochemistry. J Am Chem Soc, 2013, 135: 11562-11571

33 Ghorpade UV, Suryawanshi MP, Shin SW, et al. Eutectic solventmediated selective synthesis of $\mathrm{Cu}-\mathrm{Sb}-\mathrm{S}$-based nanocrystals: Combined experimental and theoretical studies toward highly efficient water splitting. J Mater Chem A, 2018, 6: 19798-19809

34 Chen $\mathrm{K}$, Zhou J, Chen W, et al. A green synthesis route for the phase and size tunability of copper antimony sulfide nanocrystals with high yield. Nanoscale, 2016, 8: 5146-5152

35 Chen $\mathrm{K}$, Zhou J, Chen $\mathrm{W}$, et al. Size-dependent synthesis of $\mathrm{Cu}_{12} \mathrm{Sb}_{4} \mathrm{~S}_{13}$ nanocrystals with bandgap tunability. Part Part Syst Charact, 2015, 32: 999-1005

36 Liu Y, Chen Q, Mei A, et al. Bandgap aligned $\mathrm{Cu}_{12} \mathrm{Sb}_{4} \mathrm{~S}_{13}$ quantum dots as efficient inorganic hole transport materials in planar perovskite solar cells with enhanced stability. Sustain Energy Fuels, 2019, 3: 831-840

37 Liu Y, Wang $\mathrm{H}$, Chen $\mathrm{K}$, et al. Acidic site-assisted ammonia sen- 
sing of novel $\mathrm{CuSbS}_{2}$ quantum dots/reduced graphene oxide composites with an ultralow detection limit at room temperature. ACS Appl Mater Interfaces, 2019, 11: 9573-9582

38 Liu Y, Sang B, Wang H, et al. High ammonia sensitive ability of novel $\mathrm{Cu}_{12} \mathrm{Sb}_{4} \mathrm{~S}_{13}$ quantum dots@reduced graphene oxide nanosheet composites at room temperature. Chin Chem Lett, 2020, 31: $2109-2114$

39 Fox GR, Krupanidhi SB. Nonlinear electrical properties of leadlanthanum-titanate thin films deposited by multi-ion-beam reactive sputtering. J Appl Phys, 1993, 74: 1949-1959

40 Su D, Gu C, Guo X. Functional molecular electronic devices through environmental control. Sci China Mater, 2019, 62: 1-7

41 Zhu X, Lee J, Lu WD. Iodine vacancy redistribution in organicinorganic halide perovskite films and resistive switching effects. Adv Mater, 2017, 29: 1700527

42 Bube RH. Trap density determination by space-charge-limited currents. J Appl Phys, 1962, 33: 1733-1737

43 Crowell CR, Sze SM. Current transport in metal-semiconductor barriers. Solid-State Electron, 1966, 9: 1035-1048

Acknowledgements This work was supported by the National Natural Science Foundation of China (51572205, 11674258 and 51802093), the Joint Fund of Ministry of Education for Equipment Pre-research the Fundamental Research (6141A02022262), the Excellent Dissertation Cultivation Funds of Wuhan University of Technology (2018-YS-001) and the Fundamental Research Funds for the Central Universities (2019zy-007).

Author contributions Wang Z and Liu Y, Shen J and Chen W, Miao J and Zhou J designed the experiment. Wang Z, Li A and Liu K prepared the CAS QDs, characterized the SEM, AFM, XRD, EDS, electrical features of all the films and theoretical calculation of all devices. Wang $Z$ and Liu Y prepared the manuscript. Chen W, Miao J and Zhou J helped revise the manuscript. The final version of the manuscript was approved by all authors.

Conflict of interest The authors declare that they have no conflict of interest.

Supplementary information online version of the paper.

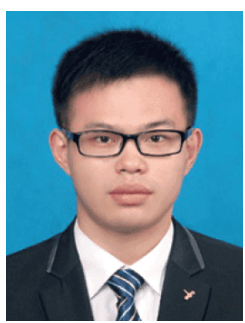

Zhiqing Wang is a master student in the School of Material Science and Engineering, Wuhan University of Technology (WUT). He obtained his bachelor's degree in materials science and engineering in June 2017 from WUT, China. His research interest is the application of quantum dot materials as resistive-switching materials for memory devices.

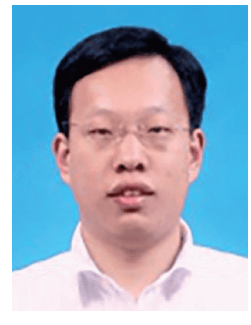

Yueli Liu is a professor in the State Key Laboratory of Silicate Materials for Architectures and School of Material Science and Engineering, WUT. He received his $\mathrm{PhD}$ degree in materials physics and chemistry in 2006 from Wuhan University, China. His research interests lie in photocatalytic and photovoltaic devices, gas sensors, metal oxide nanomaterials and quantum dots.

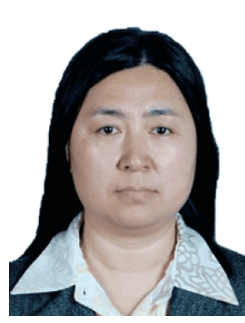

Jing Zhou is a professor of materials science and engineering at the State Key Laboratory of Advanced Technology for Materials Synthesis and Processing and School of Material Science and Engineering, WUT. She received her $\mathrm{PhD}$ degree in materials science in 2005 from WUT, China. Her research interests lie in the preparation, structure and properties of functional materials.

\section{具有尺寸依赖性的全无机 $\mathrm{Cu}_{12} \mathrm{Sb}_{4} \mathrm{~S}_{13}$ 量子点的阻 变存储性能}

王志青 ${ }^{1 \dagger}$, 刘日利 $^{2 \dagger}$, 沈杰 $^{1}$, 陈文 ${ }^{1}$, 苗君 $^{3}$, 李昂 ${ }^{1}$, 刘珂 $^{1}$, 周静 $^{1^{*}}$

摘要 全无机零维 $\mathrm{Cu}_{12} \mathrm{Sb}_{4} \mathrm{~S}_{13}$ 量子点因其优异的光学性能、带隙可 调性和载流子迁移率而受到广泛关注. 本文首次报道了 $\mathrm{Cu}_{12} \mathrm{Sb}_{4} \mathrm{~S}_{13}$ 量子点的阻变存储特性, 构建的 $\mathrm{FTO} / \mathrm{Cu}_{12} \mathrm{Sb}_{4} \mathrm{~S}_{13}$ QDs/Au阻变存储 器具有良好的可再现性和可靠的双极性电阻开关特性. 量子点的 写入/擦除电压具有尺寸依赖性, 通过控制 $\mathrm{Cu}_{12} \mathrm{Sb}_{4} \mathrm{~S}_{13}$ 量子点的尺寸 调控其能带结构进而调控写入/擦除电压, 实现了器件的写入/擦除 电压的有效调控. 同时, 器件具有较大开/关比 (大于 $10^{4}$ ), 优异的保 持性和耐久性. 在保持 $1.4 \times 10^{6} \mathrm{~s}$ 和经过 $10^{4}$ 次快速读取后, 阻变性能 变化率小于 $0.1 \%$. 性能稳定性的提升也得益于更加均一的量子点 尺寸, 因此可通过制备尺寸均匀的量子点来提升器件稳定性. 理论 计算表明 $\mathrm{Cu}_{12} \mathrm{Sb}_{4} \mathrm{~S}_{13}$ 量子点的阻变机理由空间电荷限制电流机制占 主导, 在电场作用下, $\mathrm{Cu} 3 \mathrm{~d} 、 \mathrm{Cu} 3 \mathrm{p}$ 和 $\mathrm{S} 3 \mathrm{p}$ 作为电子自俘获中心进 行电子的俘获与去俘获, 从而改变了 $\mathrm{Cu}_{12} \mathrm{Sb}_{4} \mathrm{~S}_{13}$ 量子点的阻值. 结果 表明, $\mathrm{Cu}_{12} \mathrm{Sb}_{4} \mathrm{~S}_{13}$ 量子点可作为一种新型的阻性开关材料, 在数据存 储器件中具有巨大的应用潜力, 有望促进下一代非易失性存储器 的发展与应用. 